\title{
Photon-photon correlations and entanglement in doped photonic crystals
}

\author{
David Petrosyan and Gershon Kurizki \\ Department of Chemical Physics, Weizmann Institute of Science, Rehovot 76100, Israel
}

(November 20, 2018)

\begin{abstract}
We consider a photonic crystal (PC) doped with four-level atoms whose intermediate transition is coupled near-resonantly with a photonic band-gap edge. We show that two photons, each coupled to a different atomic transition in such atoms, can manifest strong phase or amplitude correlations: One photon can induce a large phase shift on the other photon or trigger its absorption and thus operate as an ultrasensitive nonlinear photon-switch. These features allow the creation of entangled two-photon states and have unique advantages over previously considered media: (i) no control lasers are needed; (ii) the system parameters can be chosen to cause full two-photon entanglement via absorption; (iii) a number of PCs can be combined in a network.

PACS number(s): 42.50.Gy, 03.67.-a, 42.70.Qs
\end{abstract}

\section{INTRODUCTION}

Nonlinear effects whereby one light beam influences another require large numbers of photons [1] or else photon confinement in a high-Q cavity [2]. Hence the impediment towards constructing quantum logical gates operating at the few-photon level. The ingenious attempt to achieve increased photon-photon coupling in a gas by means of control laser fields [3] has resulted in cooperatively enhanced single-photon absorption and emission (as for excitons in solids), but not in two-photon entanglement [4]. A promising avenue has been opened by studies of enhanced nonlinear coupling via electromagnetically induced transparency (EIT) in gases in the presence of control laser fields, which induce coherence between atomic levels $[5$. These studies have predicted the ability to achieve an appreciable nonlinear phase shift using extremely weak optical fields or a two-photon switch in the $N$-configuration of atomic levels [6.7]. Further improvement of the sensitivity of these schemes has been suggested using a rather involved system, in which a second species of coherently driven $\Lambda$-atoms provides the matching of the group velocities of interacting photons [8].

Here we point out that photon-photon nonlinear phase shifters and switches are realizable with a potentially very high efficiency and without external laser fields in photonic crystals (PCs) 19 11] dilutely doped with fourlevel atoms. These atoms have two transitions tuned to the two incident photons and an intermediate transition tuned to a singular feature of the structured density of modes (DOM) spectrum of the PC. The strong nonlinear effects analyzed in Secs. II and III arise from the coherent Autler-Townes splitting of atomic emission lines near a photonic band-gap (PBG) edge 12 15 and the strong photon-atom coupling via photonic defect modes in the doped PC [16, 17]. Transparency near a band edge, which has been previously predicted for an atomic threelevel $\Lambda$-configuration [18], is shown here (Sec. III) to be most suitable for two-photon absorption switching. This mechanism, which has not been studied in PCs, is demonstrated to be considerably more efficient than its coun- terpart in Ref. [7]. It is predicted to allow complete absorption of one photon in the presence of another photon, and thereby the creation of a fully entangled two-photon state (Sec. IV]). By contrast, appreciable (but limited) nonlinear phase-shifting is shown (Sec. IIII) to arise by tuning the photon frequency to the Raman resonance with the defect mode frequency associated with atomic doping. These features have unique advantages for quantum information applications (Sec. IV), as compared to previously considered media.

\section{THEORY}

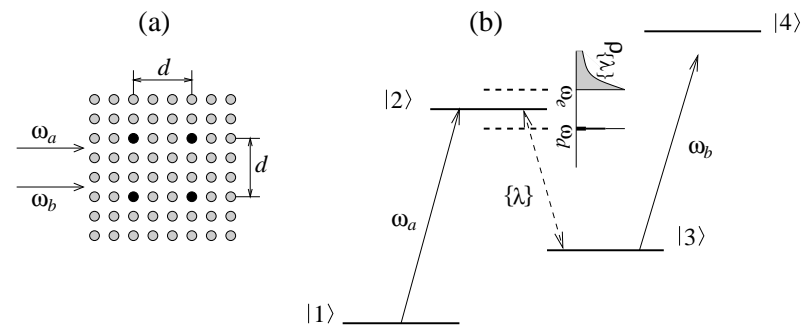

FIG. 1. (a) Photonic crystal dilutely doped with atoms located at black dots. (b) Four-level atom coupled to a structured continuum $\{\lambda\}$ near the band-edge or defect mode frequencies (DOM plotted) via the intermediate transition $|2\rangle \rightarrow|3\rangle$ and interacting with two photons $\omega_{a}$ and $\omega_{b}$ at the sideband transitions $|1\rangle \rightarrow|2\rangle$ and $|3\rangle \rightarrow|4\rangle$, respectively.

We examine the nonlinear coupling of two optical fields $\hat{E}_{a}=f_{a} \varepsilon_{a} \hat{a}$ and $\hat{E}_{b}=f_{b} \varepsilon_{b} \hat{b}$, where $\hat{a}$ and $\hat{b}$ are the respective annihilation operators, $\varepsilon_{i}=\left(\hbar \omega_{i} / 2 \epsilon_{0} V_{i}\right)^{1 / 2}$ is the field amplitude per photon $\omega_{i}(i=a, b)$ within the quantization volume $V_{i}=\sigma_{i} l_{i}$, and $f_{i}(z, t)$ is the singlephoton wavepacket envelope. The two fields propagate along the $z$-axis in a PC dilutely doped with identical four-level atoms. The level configuration of the atom and the DOM are depicted in Fig. 11, where the unperturbed atomic levels $|j\rangle, j=1 \ldots 4$, have the corresponding energies $\hbar \omega_{j}$. The incident photons at frequencies $\omega_{a}$ and $\omega_{b}$ 
interact with the atoms via the transitions $|1\rangle \rightarrow|2\rangle$ and $|3\rangle \rightarrow|4\rangle$, respectively, while the transition $|2\rangle \rightarrow|3\rangle$ is coupled to the structured PC mode-continuum $\{\lambda\}$. In the dipole and rotating-wave approximations, the Hamiltonian of the $l$ th atom +field can be written as

$$
\begin{aligned}
H^{(l)}= & \sum_{j} \hbar \omega_{j} \hat{\sigma}_{j j}^{(l)}-\hbar\left[\sum_{\lambda} g_{\lambda} \hat{c}_{\lambda} e^{i\left(k_{\lambda} z-\omega_{\lambda} t\right)} \hat{\sigma}_{23}^{(l)}\right. \\
& \left.+g_{a} \hat{a} e^{i\left(k_{a} z-\omega_{a} t\right)} \hat{\sigma}_{21}^{(l)}+g_{b} \hat{b} e^{i\left(k_{b} z-\omega_{b} t\right)} \hat{\sigma}_{43}^{(l)}+\text { H. c. }\right],
\end{aligned}
$$

where $k_{i}(i=a, b, \lambda)$ is the wave number of the corresponding mode, $g_{i}=\mu_{j k} f_{i} \varepsilon_{i} / \hbar$ its atom-field coupling strength, $\mu_{j k}$ being the dipole matrix element for the atomic transition $|j\rangle \rightarrow|k\rangle, \hat{\sigma}_{j k}^{(l)}=|j\rangle\langle k|$ is the atomic operator, $\hat{c}_{\lambda}$ is the $\lambda$ mode annihilation operator and $\sum_{\lambda} \rightarrow \int d \omega_{\lambda} \rho\left(\omega_{\lambda}\right)$, where $\rho\left(\omega_{\lambda}\right)$ is the DOM of the structured continuum.

We assume that initially the two incident photons are in the product state $\left|1_{a}\right\rangle\left|1_{b}\right\rangle$, the atoms are in the ground state $|1\rangle$ and the continuum is in the vacuum state $\left|0_{\lambda}\right\rangle$. Then the wave function of the system reads $\left|\Phi\left(z_{l}, t\right)\right\rangle=A_{1}\left|1,0_{\lambda}, 1_{a}, 1_{b}\right\rangle+A_{2}\left|2,0_{\lambda}, 0_{a}, 1_{b}\right\rangle+$ $\sum_{\lambda} A_{3, \lambda}\left|3,1_{\lambda}, 0_{a}, 1_{b}\right\rangle+\sum_{\lambda} A_{4, \lambda}\left|4,1_{\lambda}, 0_{a}, 0_{b}\right\rangle$. With the Hamiltonian (11), the Schrödinger equation leads to the following set of equations for the slowly-varying (during an optical cycle) probability amplitudes $A_{j}$ :

$$
\begin{aligned}
\frac{\partial A_{1}}{\partial t} & =i \Omega_{a}^{*} A_{2} \\
\frac{\partial A_{2}}{\partial t} & =\left[i \Delta_{a}-\gamma_{2}\right] A_{2}+i \Omega_{a} A_{1}+i \int d \omega_{\lambda} \rho\left(\omega_{\lambda}\right) g_{\lambda} A_{3, \lambda} \\
\frac{\partial A_{3, \lambda}}{\partial t} & =\left[i\left(\Delta_{a}-\Delta_{\lambda}\right)-\gamma_{3}\right] A_{3, \lambda}+i g_{\lambda}^{*} A_{2}+i \Omega_{b}^{*} A_{4, \lambda} \\
\frac{\partial A_{4, \lambda}}{\partial t} & =\left[i\left(\Delta_{a}-\Delta_{\lambda}+\Delta_{b}\right)-\gamma_{4}\right] A_{4, \lambda}+i \Omega_{b} A_{3, \lambda}
\end{aligned}
$$

(2a)is the linear resonant absorption coefficient on the atomic (2b) cross-section, $N$ the density of doping atoms and $n_{a}$ the

$$
I=\int \frac{d \omega_{\lambda} \rho\left(\omega_{\lambda}\right)\left|g_{\lambda}\right|^{2}}{\gamma_{3}-i\left(\Delta_{a}-\Delta_{\lambda}\right)+\left|\Omega_{b}\right|^{2}\left[\gamma_{4}-i\left(\Delta_{a}-\Delta_{\lambda}+\Delta_{b}\right)\right]^{-1}}
$$

where $\Omega_{i}=\mu_{j k}\left\langle 0_{i}\left|\hat{E}_{i}\right| 1_{i}\right\rangle / \hbar=g_{i}$ is the Rabi frequency of the corresponding field at the position of the $l$ th atom, $\Delta_{a}=\omega_{a}-\omega_{21}, \Delta_{b}=\omega_{b}-\omega_{43}$ and $\Delta_{\lambda}=\omega_{\lambda}-\omega_{23}$ are the detunings from the respective atomic transition frequencies, and $\gamma_{j}(j=2,3,4)$ is the relaxation rate of level $|j\rangle$, which accounts for both spontaneous radiative decay at a frequency far from PBG so that it is treated as a Markovian process (Wigner-Weisskopf approximation) and nonradiative (mainly vibrational) relaxation in the PC.

The Wigner-Weisskopf approximation is inapplicable near the PBG where the DOM varies rapidly [12]. Thus, in the vicinity of the transition frequency $\omega_{23}$, we must exactly integrate the last term of Eq. (2b) for the specific PBG model employed. To solve Eqs. (2), we make the weak-field approximation $A_{1} \simeq 1$ (much less than one photon per atom) and use the second-order perturbation theory thus obtaining the steady-state expressions for the atomic response. In doing so, one can see that the probability amplitude $A_{4, \lambda}$ is inversely proportional to the detuning $\Delta_{b}$. Then, near the Raman resonance transition $|1\rangle \rightarrow|2\rangle$, with $\sigma_{0}$ the resonant absorption (averaged) refraction index for the $\omega_{a}$ photon, and

$\Delta_{a} \simeq \Delta_{\lambda}$, the right-hand side of Eq. (2d) contains a term $\gamma_{4}\left|\Omega_{b}\right|^{2} /\left(\Delta_{b}^{2}+\gamma_{4}^{2}\right)$ resulting in an additional relaxation of the amplitude $A_{3, \lambda}$ and thus destroying the coherence between levels $|1\rangle$ and $|3\rangle$ which induces the absorption of the $\omega_{a}$ photon. To minimize this decoherence, we take $\left|\Delta_{b}\right| \gg\left|\Omega_{b}\right|, \gamma_{4}$, so that the depletion of $\Omega_{b}$ can safely be neglected. In the opposite case of small detunng $\left|\Delta_{b}\right| \leq \gamma_{4}$, it has been suggested to use this effect for structing a sensitive two-photon switch [7].

Under these conditions, one obtains effectively freespace propagation of the $\hat{E}_{b}$ field. By contrast, the Rabi frequency of the $\hat{E}_{a}$ field, in the slowly varying envelope approximation, obeys the following propagation equation

$$
\left[\frac{\partial}{\partial z}+\frac{1}{v_{g}} \frac{\partial}{\partial t}\right] \Omega_{a}=i \alpha \Omega_{a}
$$

with the solution $\Omega_{a}(z, t)=\Omega_{a}\left(0, t-z / v_{g}\right) \exp (i \alpha z)$. Here the macroscopic complex polarizability $\alpha$ is given, under the weak-field linear-response assumption, by

$$
\alpha=\alpha_{0} \frac{i \gamma_{2}}{\gamma_{2}-i \Delta_{a}+I}
$$

is the integral of the saturation factor over the structured DOM. The group velocity $v_{g}$ is expressed as

$$
v_{g}=\frac{\partial \omega_{a}}{\partial k_{a}}=\left[\frac{n_{a}}{c}+\frac{\partial \operatorname{Re}(\alpha)}{\partial \omega_{a}}\right]^{-1} .
$$

At the exit from the medium $z=\zeta$, the delay time $T_{\text {del }}$ of the field, relative to the passage time $T_{0}=\zeta n_{a} / c$ through a passive medium, can be written as

$$
T_{\mathrm{del}}=\frac{\partial \operatorname{Re}(\alpha)}{\partial \omega_{a}} \zeta
$$

The slowly varying field-propagation equation (3) must be justified upon examining the group-velocity dispersion

$$
D=\frac{\partial^{2} k_{a}}{\partial \omega_{a}^{2}}=\frac{\partial^{2} \operatorname{Re}(\alpha)}{\partial \omega_{a}^{2}}
$$

which is responsible for the spreading and reshaping of the photon pulse. In analogy with the weakly interacting 
Bose gas, $D$ can be interpreted as being inversely proportional to the photon "mass". In what follows, conditions such that $D$ is small are discussed for $\omega_{a}$. Due to the weak-field approximation $A_{1} \simeq 1$ and large detuning $\Delta_{b}$, the $\omega_{b}$ photon propagation is nearly free and its group velocity dispersion is negligible.

To calculate integral (5), we assume the isotropic PBG model [12 15] with the atoms doped at the positions of the local defects in the PC separated by a distance $d$ from each other. These "impurities" of the crystal structure form the defect modes in the PBG, which are localized at each atomic site in a volume $V_{d} \simeq(r L)^{3}$ of several $(r)^{3}$ lattice cells $L^{3}$ 11, 16, 17. In the dilute regime $d>r L$, one can neglect dipole-dipole interactions and tunneling ("hopping") of photons between the atoms 13, 19 and the defect modes can serve as high-Q cavities. For $\omega_{23}$ near (or within) the $\mathrm{PBG}$ frequency, we have $L \simeq \pi c / \omega_{23}$. Hence, the dilute regime limits the dopant density to $N<\left(\omega_{23} / \pi c r\right)^{3}$. Then, in the vicinity of the upper edge $\omega_{e}$ of the PBG, the DOM function can be written as

$$
\rho(\omega)=\rho_{d} \delta\left(\omega-\omega_{d}\right)+\rho_{e} \frac{\Theta\left(\omega-\omega_{e}\right)}{\sqrt{\omega-\omega_{e}}},
$$

where $\Theta(\omega)$ is the Heaviside step function, $\rho_{d}$ and $\rho_{e}$ are PC-specific constants [12,15], and $\omega_{d}$ is the frequency of the defect mode. The integration of Eq. (5), with $\rho\left(\omega_{\lambda}\right)$ given by Eq. (9) and $\Delta_{b} \gg \Delta_{a, \lambda}$, leads to

$$
I=\frac{\beta_{d}^{2}}{\gamma_{31}-i\left(\Delta_{a}-\Delta_{d}-s_{3}\right)}-\frac{\beta_{e}^{3 / 2}}{\sqrt{i \gamma_{31}+\left(\Delta_{a}-\Delta_{e}-s_{3}\right)}},
$$

where $\Delta_{d, e}=\omega_{d, e}-\omega_{23} \ll \omega_{23}$ are the detunings of the defect-mode and PBG-edge frequencies from the atomic resonance $\omega_{23}, \gamma_{31}=\gamma_{3}+\gamma_{4}\left|\Omega_{b}\right|^{2} / \Delta_{b}^{2}$ the $|1\rangle \leftrightarrow|3\rangle$ decoherence rate, $s_{3}=\left|\Omega_{b}\right|^{2} / \Delta_{b}$ the ac Stark shift of level $|3\rangle$, and 1215

$$
\beta_{d}^{2}=\frac{\left|\mu_{23}\right|^{2} \omega_{23}^{4}}{2 \epsilon_{0} \hbar(\pi c r)^{3}}, \quad \beta_{e}^{3 / 2}=\frac{\left|\mu_{23}\right|^{2} \omega_{23}^{7 / 2}}{6 \epsilon_{0} \hbar \pi c^{3}}
$$

are the coupling constants of the atom with the structured reservoir, whose main contributions are near $\omega_{d}$ and $\omega_{e}$.

\section{RESULTS}

To illustrate the results of the foregoing analysis, we first plot in Fig. 2 the polarizability (4), delay time (7) and group-velocity dispersion (8) for the case of one incident photon $\omega_{a}\left(\Omega_{b}=0\right)$. Clearly, two frequency regions, $\Delta_{a} \sim \Delta_{d}$ and $\Delta_{a} \sim \Delta_{e}$, where the absorption vanishes and, at the same time, the dispersion slope is steep, are of particular interest. One can see in Fig. 2(a),(b), where we plot the spectrum for two different values of the coupling constants $\beta_{d}$ and $\beta_{e}$, that there is, however, a substantial difference between the spectra in the foregoing frequency regions, for the following physical reasons:
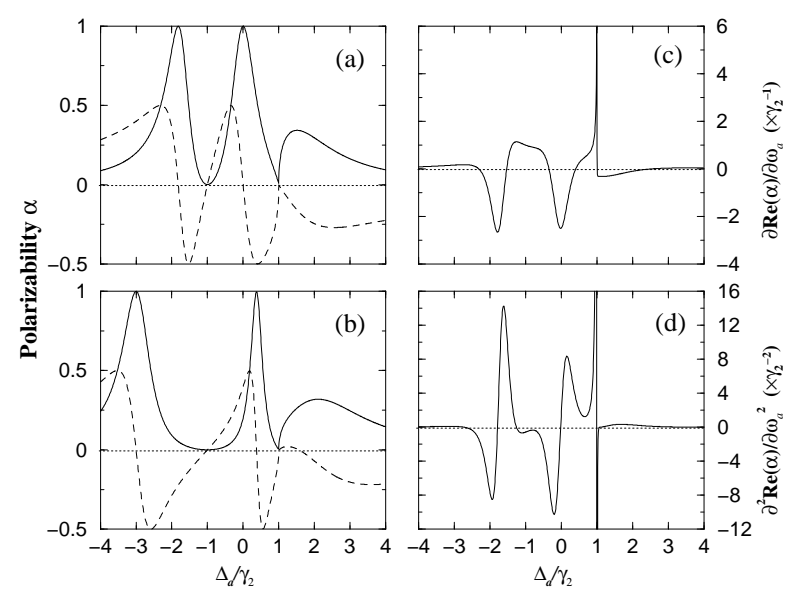

FIG. 2. (a),(b) Imaginary (solid lines) and real (dashed lines) part of the complex polarizability $\alpha$, as a function of the detuning $\Delta_{a}$ for the case $\Omega_{b}=0, \alpha_{0}=1 \mathrm{~cm}^{-1}, \Delta_{d}=-1$, $\Delta_{e}=1$ and $\gamma_{31}=0.001$. (a) $\beta_{d}=\beta_{e}=1$; (b) $\beta_{d}=\beta_{e}=2$. (c) Delay time $T_{\text {del }}$ (per unit $z$ ), and (d) group velocity dispersion coefficient $D$ as a function of the detuning $\Delta_{a}$ for the same parameters as in (a). All parameters are in units of $\gamma_{2}$.

a) In the vicinity of $\Delta_{d}$, the radiation emitted by the atom at the frequency $\omega_{d}$ remains confined in the defect mode for a long time as in a high-Q cavity. As the $\omega_{a}$ photon wave packet approaches the $l$ th atom, $\Omega_{a} / \beta_{d}(\ll 1)$ fraction of its amplitude is transferred into the defect mode, inducing the corresponding population of level $|3\rangle$ (assuming adiabatic Raman transfer [20]). At the end of the wavepacket, when $\Omega_{a} \rightarrow 0$, all the population of level $|3\rangle$ returns to $|1\rangle$ and the radiation that has been confined to the defect mode is added to the tail of the propagating wave packet, until it encounters the next atom. This strong interaction of the atom with the defect mode at $\Delta_{a} \simeq \Delta_{d}$ splits the spectrum by the amount equal roughly to $2 \beta_{d}$ and causes EIT [5]. The transparency window is rather broad and is given by the inverse Lorentzian [see the first term on the right-hand side of Eq. (10)]. The corresponding group velocity is much smaller than the speed of light:

$$
v_{g} \simeq\left[\frac{\partial \operatorname{Re}(\alpha)}{\partial \omega_{a}}\right]^{-1} \sim \frac{\beta_{d}^{2}}{\gamma_{2} \alpha_{0}} \ll c,
$$

which leads to a large delay time $T_{\mathrm{del}}=\zeta / v_{g}$ [Fig. 2(c)]. One has to keep in mind, however, that the absorptionfree propagation time is limited by the EIT decoherence time $T_{\text {del }}<\gamma_{31}^{-1}$ [5], which imposes a limitation on the length $\zeta$ of the active PC medium. The corresponding group-velocity dispersion (8) is small (a large photon mass), and, therefore, will not cause much spread of the $\omega_{a}$ wave packet [Fig. 2(d)]. 
b) In the vicinity of $\Delta_{e}$, the strong interaction of the atom with the continuum near the band edge $\omega_{e}$ causes the Autler-Townes splitting of level $|2\rangle$ into a doublet with a separation equal roughly to $\beta_{e}$. One component of this doublet is shifted out of the PBG while the other one remains within the gap and forms the so called photon-atom bound state 12 15, 18. Consequently, there is vanishing absorption and rapid variation of the dispersion at $\Delta_{a} \simeq \Delta_{e}$. Here the delay time can be large, but the group velocity dispersion is also very large [Fig. 2(c),(d)]. Hence, as $\omega_{a}$ is tuned very close to the band edge, the transmitted pulse shape is distorted. Since the transparency region is very narrow with a width $\Delta \omega \sim \gamma_{3}\left(\ll \gamma_{2}, \beta_{e}\right)$, for an absorption-free propagation of the $\omega_{a}$ photon, the temporal width of its wavepacket $\tau_{a}=l_{a} / c$ should satisfy the condition $\tau_{a}>\pi / \Delta \omega$. Simultaneously, a small deviation from the condition $\Delta_{a}=\Delta_{e}$ will lead to a strong increase in the absorption of the $E_{a}$ field.
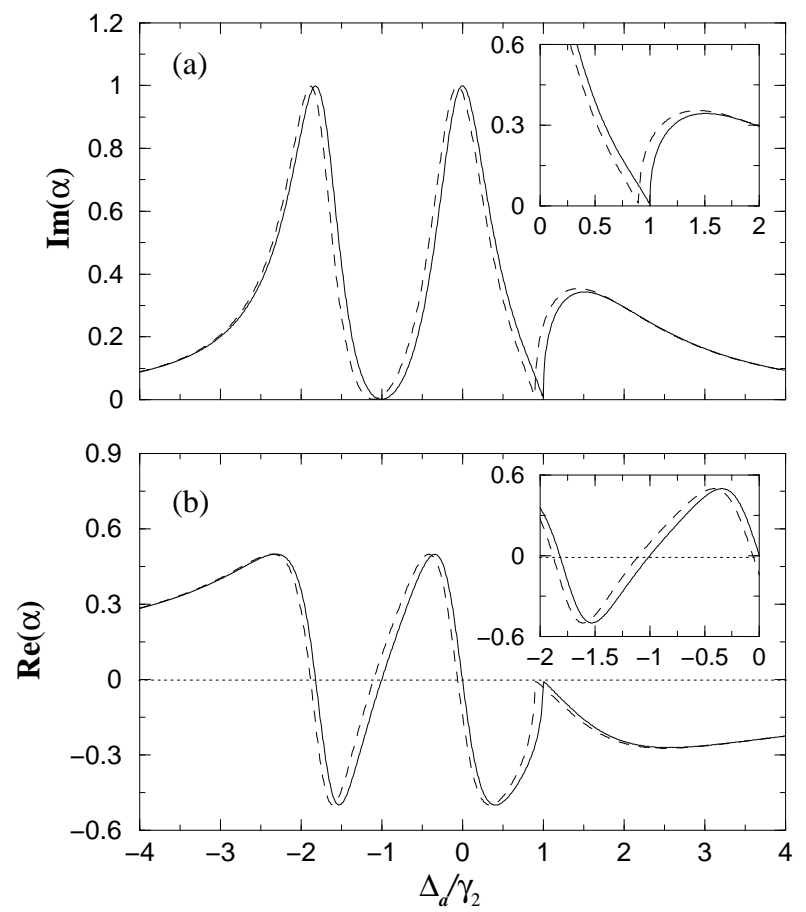

FIG. 3. (a) Imaginary, and (b) real part of the complex polarizability $\alpha$ as a function of the detuning $\Delta_{a}$ for the case $\Omega_{b}=0$ (solid lines) and $\Omega_{b}=g_{b}$ (dashed lines). All parameters are the same as in Fig. 2 (a) and $s_{3}=-0.1$ (i.e., $\left.\Delta_{b}=-10\left|\Omega_{b}\right|^{2}\right)$. The insets magnify the important frequency regions.

Let us now switch on the $\omega_{b}$ photon. As seen from Eq. (10), its effect is merely to shift the spectrum by the amount equal to $s_{3}$ (Fig. 3). This shift, however, will have different implications in the two frequency regions distinguished above:

i) If $s_{3} \ll \beta_{d}$, i.e., the Stark shift is smaller than the width of the transparency window at $\Delta_{d}$, the medium will still remain transparent for a $\omega_{a}$ photon with the detuning $\Delta_{a}=\Delta_{d}$, but its phase will experience an appreciable nonlinear shift $\phi_{a}$ given by

$$
\phi_{a}=\operatorname{Re}(\alpha) z \simeq-\frac{\partial \operatorname{Re}(\alpha)}{\partial \omega_{a}} s_{3} z \sim-\frac{\gamma_{2} \alpha_{0}}{\beta_{d}^{2}} s_{3} z .
$$

ii) On the other hand, for a $\omega_{a}$ photon with the detuning $\Delta_{a}=\Delta_{e}$, the medium, which is transparent for $\Omega_{b}=0$, having

$$
\operatorname{Im}(\alpha) \simeq \frac{\gamma_{2} \alpha_{0}}{\beta_{e}^{3 / 2}} \sqrt{\frac{\gamma_{3}}{2}} \ll \alpha_{0},
$$

will become highly absorptive (opaque) even for such a small frequency shift as $s_{3}$ (provided $\left|s_{3}\right|>\Delta \omega$ ), changing (13) to

$$
\operatorname{Im}(\alpha) \simeq\left\{\begin{array}{ll}
\frac{\gamma_{2}^{2} \alpha_{0}}{\beta_{e}^{3}} s_{3}, & s_{3}>0 \\
\frac{\gamma_{2} \alpha_{0}}{\beta_{e}^{3 / 2}} \sqrt{\left|s_{3}\right|}, & s_{3}<0
\end{array},\right.
$$

and thus acting as an ultrasensitive, effective switch. These are the main results of the present work.

The remaining question is how to maximize the interaction between the $\omega_{a}$ photon, which propagates with a small group velocity (11), and the $\omega_{b}$ photon, which propagates with a velocity close to the speed of light. A possible technique to achieve equal group velocities for both photons, as suggested in Ref. [8], is to have a second kind of $\Lambda$-atoms in the interaction region and apply a driving field that would result in EIT and reduced group velocity for the $\omega_{b}$ photon. To keep our scheme simple, we have chosen here not to adopt a similar approach.

In our system, the interaction between the photons is maximized if: they enter the medium simultaneously; the transverse shapes of their tightly focused $\left(\sigma_{a, b} \sim \sigma_{0}\right)$ wavepackets overlap completely; and the quantization (wavepacket) length $l_{b}$ of the $\omega_{b}$ photon satisfies the condition $\left(l_{b}+\zeta\right) / c \leq \zeta / v_{g}$. Then the $\omega_{b}$ photon leaves the medium not later than the $\omega_{a}$ photon. The effective interaction length between the two photons is therefore $z_{\text {eff }} \sim l_{b} v_{g} / c \leq \zeta$, after which the two wavepackets slip apart. This effective interaction length has the following implications:

1) Since $s_{3} \propto 1 / l_{b}$, the interaction-induced phase shift (12) saturates with distance and is the same for all $l_{b}$ satisfying the above condition:

$$
\phi_{a}=-\frac{\partial \operatorname{Re}(\alpha)}{\partial \omega_{a}} s_{3} \frac{l_{b} v_{g}}{c} \simeq-\frac{\left|\mu_{34}\right|^{2} \omega_{b}}{2 \epsilon_{0} \hbar c \sigma_{b} \Delta_{b}} .
$$

2) As was mentioned in Sec. II and carefully analyzed in Ref. [7], in the case of a small detuning $\left|\Delta_{b}\right| \leq \gamma_{4}$, the presence of the $\omega_{b}$ photon will result in the destruction of EIT in the vicinity of $\Delta_{a}=\Delta_{d}$ and, consequently, the strong absorption, which is determined by

$$
\operatorname{Im}(\alpha) \simeq \frac{\gamma_{2} \alpha_{0}\left|\Omega_{b}\right|^{2}}{\gamma_{4} \beta_{d}^{2}} .
$$


With the effective interaction length $z_{\text {eff }} \sim l_{b} v_{g} / c$, where $v_{g}$ is given by Eq. (11), the power loss at the exit from the $\mathrm{PC}$ is

$$
2 \operatorname{Im}(\alpha) z_{\mathrm{eff}} \simeq \frac{\left|\mu_{34}\right|^{2} \omega_{b}}{\epsilon_{0} \hbar c \sigma_{b} \gamma_{4}}
$$

Thus, in the vicinity of the two-photon (Raman) resonance with the defect mode, $\Delta_{a}=\Delta_{d}$, the absorption (16) $\left(\left|\Delta_{b}\right| \leq \gamma_{4}\right)$, as well as the phase shift (15) $\left(\left|\Delta_{b}\right| \gg\left|\Omega_{b}\right|, \gamma_{4}\right)$, saturate over a distance equal to $z_{\text {eff. }}$

3) The dramatic advantage of the present scheme over conventional EIT schemes [6,7] is that, in the vicinity of the two-photon resonance with the band edge frequency, $\Delta_{a}=\Delta_{e}$, the group velocity of the $\omega_{a}$ photon wavepacket is close to the speed of light in the presence of the nearlyfree propagating $\omega_{b}$ photon wavepacket [see Fig. 2(c)]. Therefore, there is no velocity mismatch of the two photons and no saturation of the absorption with distance, the interaction length being close to the length $\zeta$ of the PC. Thus the absorption probability of the $\omega_{a}$ photon, $1-\exp [-2 \operatorname{Im}(\alpha) \zeta]$, where $\operatorname{Im}(\alpha)$ is given by (14), can be made arbitrary close to unity by choosing a long enough PC.

It can be checked that, for the parameter values used in Fig. 3, and $\mu_{34} \sim 1$ a.u., $\omega_{b}=4 \times 10^{15} \mathrm{rad} / \mathrm{s}\left(\lambda_{b}=470\right.$ $\mathrm{nm}),\left|\Delta_{b}\right|=10 \gamma_{2}, \gamma_{2} \sim 5 \times 10^{7} \mathrm{~s}^{-1}, \sigma_{b} \sim 10^{-10} \mathrm{~cm}^{-2}$, we obtain the power $\operatorname{loss} 2 \operatorname{Im}(\alpha) \zeta \sim 0.46 \zeta$ at $\Delta_{a}=\Delta_{e}$ and the phase shift $\phi_{a} \sim 0.1 \mathrm{rad}$ at $\Delta_{a}=\Delta_{d}$. Thus, the presence of one $\omega_{b}$ photon induces either strong absorption or a large phase shift of the $\omega_{a}$ photon, depending on the frequency region employed.

\section{DISCUSSION}

The foregoing results have demonstrated the ultrahigh sensitivity of photonic absorption or phase shift in a doped PC to the presence of an additional photon. Using the procedure of Ref. [7] one can employ these features to construct two types of entanglement between the photons $\omega_{a}$ and $\omega_{b}$ :

a) Suppose that a photon pair $|1\rangle_{\omega_{a}}|1\rangle_{\omega_{b}}$ is simultaneously generated in some parametric or other process. The $\omega_{a}$ photon enters the doped PC. The $\omega_{b}$ photon is split between the two arms of a $50 \%-50 \%$ beam splitter, resulting in an entangled state $1 / \sqrt{2}\left(|10\rangle_{\omega_{b}}+|01\rangle_{\omega_{b}}\right)$. One arm of this state, say $|10\rangle_{\omega_{b}}$, enters the PC together with the $\omega_{a}$ photon, while the other arm, $|01\rangle_{\omega_{b}}$, does not. In the case of the $\omega_{a}$ photon with the detuning $\Delta_{a} \simeq \Delta_{e}$, after passing through the $\mathrm{PC}$, the resulting state of the system has the fully entangled form

$$
\begin{aligned}
& \frac{1}{\sqrt{2}}\left(|10\rangle_{\omega_{b}}+|01\rangle_{\omega_{b}}\right) \otimes|1\rangle_{\omega_{a}} \\
& \rightarrow \frac{1}{\sqrt{2}}\left(|10\rangle_{\omega_{b}}|0\rangle_{\omega_{a}}+|01\rangle_{\omega_{b}}|1\rangle_{\omega_{a}}\right),
\end{aligned}
$$

wherein the states in which the $\omega_{a}$ photon is or is not absorbed are equally superposed.

$b)$ In the case of the $\omega_{a}$ photon being initially in a state with a rather well defined phase (i.e., coherent state) $|\alpha\rangle$ and having the detuning $\Delta_{a} \simeq \Delta_{d}$, after passing through the $\mathrm{PC}$, the resulting state of the system is given by

$$
\begin{aligned}
& \frac{1}{\sqrt{2}}\left(|10\rangle_{\omega_{b}}+|01\rangle_{\omega_{b}}\right) \otimes|\alpha\rangle_{\omega_{a}} \\
& \rightarrow \frac{1}{\sqrt{2}}\left(|10\rangle_{\omega_{b}}\left|e^{i \phi_{a}} \alpha\right\rangle_{\omega_{a}}+|01\rangle_{\omega_{b}}|\alpha\rangle_{\omega_{a}}\right),
\end{aligned}
$$

wherein the states in which the $\omega_{a}$ photon does or does not acquire the phase shift $\phi_{a}$ are equally superposed. The state (18) is fully entangled only if $\left|e^{i \phi_{a}} \alpha\right\rangle$ and $|\alpha\rangle$ do not overlap in the phase plane (the same requirement as for a "Schrödinger cat" state in a PC [17]).

These features can be used to appreciably advance towards the goal of producing entangled states of radiation or logical photon switches for quantum information processing, owing to the unique advantages of the doped PCs over conventional EIT schemes [6] or high-Q cavities [2]: (i) No control lasers are needed to create the quantum interference responsible for the EIT effects. (ii) The system parameters can easily be adjusted to provide full two-photon entanglement via absorption. (iii) Several doped PCs can be combined via dispersive couplers either to enhance the accumulated nonlinear phase-shift or to perform a chain of logical operations. The fact that the band structure of dilutely doped PCs is insensitive to the locations or exact concentration of the dopants and defects should facilitate the incorporation of several PCs in one network.

\section{ACKNOWLEDGMENTS}

This work was supported by the US-Israel BSF and the Feinberg Fellowship (D.P.).

[1] N.W. Boyd, Nonlinear Optics (Academic, San Diego, CA, 1992).

[2] H.J. Kimble, Phys. Scr. 76, 127 (1998); A. Imamoğlu, H. Schmidt, G. Woods, and M. Deutsch, Phys. Rev. Lett. 79, 1467 (1997); M.J. Werner and A. Imamoğlu, Phys. Rev. A 61, 011801(R) (2000).

[3] J.D. Franson and T.B. Pittman, Phys. Rev. A 60, 917 (1999); quant-ph/9912121.

[4] T. Opatrny and G. Kurizki, Fort. der Physik 48(9), 1125 (2000); quant-ph/000301.

[5] M.O. Scully and M.S. Zubairy, Quantum Optics (Cambridge University Press, Cambridge, 1997); S.E. Harris, Phys. Today 50(7), 36 (1997). 
[6] H. Schmidt and A. Imamoğlu, Opt. Lett. 21, 1936 (1996).

[7] S.E. Harris and Y. Yamamoto, Phys. Rev. Lett. 81, 3611 (1998); S.E. Harris and L.V. Hau, ibid 82, 4611 (1999).

[8] M.D. Lukin and A. Imamoğlu, Phys. Rev. Lett. 84, 1419 (2000).

[9] E. Yablonovitch, Phys. Rev. Lett. 58, 2059 (1987).

[10] S. John, Phys. Rev. Lett. 58, 2486 (1987).

[11] J.D. Joannopoulos, R.D. Meade, and J.N. Winn, Photonic Crystals: Molding the Flow of Light (Princeton University Press, Princeton, 1995).

[12] A.G. Kofman, G. Kurizki, and B. Sherman, J. Mod. Opt. 41, 353 (1994).

[13] S. John and J. Wang, Phys. Rev. Lett 64, 2418 (1990); Phys. Rev. B 43, 12772 (1991).

[14] S. John and T. Quang, Phys. Rev. A 50, 1764 (1994).

[15] P. Lambropoulos, G. M. Nikolopoulos, T. R. Nielsen, and S. Bay, Rep. Prog. Phys. 63, 455 (2000).

[16] P.R. Villeneuve, S. Fan, and J.D. Joannopoulos, Phys. Rev. B 54, 7837 (1996); E. Yablonovitch et al., Phys. Rev. Lett. 67, 3380 (1991).

[17] B. Sherman, G. Kurizki, and A. Kadyshevitch, Phys. Rev. Lett. 69, 1927 (1992); G. Kurizki, B. Sherman, and A. Kadyshevitch, J. Opt. Soc. Am. B 10, 346 (1993).

[18] E. Paspalakis, N.J. Kylstra, and P.L. Knight, Phys. Rev. A 60, R33 (1999).

[19] S. Bay, P. Lambropoulos, and K. Mølmer, Phys. Rev. A 55, 1485 (1997).

[20] M. Fleischhauer and M. D. Lukin, Phys. Rev. Lett. 84, 5094 (2000). 